\title{
The Konar Sandal 'Geometric' inscriptions: a graphotactic analysis
}

\author{
Matthieu Kervran ${ }^{1}$ \\ mkervran_research@orange.fr
}

\begin{abstract}
An analysis of the distributional properties of the signs in the Konar Sandal 'Geometric' inscriptions ( $3^{\text {rd }}$ millenium B.C.E.) suggests that they may in fact be musical scores.
\end{abstract}

Keywords: Konar Sandal, Jiroft, Geometric writing, Linear Elamite, Archaeomusicology

The Konar Sandal 'Geometric' inscriptions are extant on three tablets found in 2005 and 2006 by an archaeological mission in the village of Konar Sandal, near the town of Jiroft in Iran. See [Basello 2006] for the publication of the first 'Geometric' inscription, and [Madjidzadeh 2012] on the circumstances of their discovery.

[Desset 2014] contains the first detailed analysis of the three inscriptions, and names them $\alpha$, $\beta$ and $\gamma$. The same names will be used in this paper to refer to the inscriptions.

The tablets are attributed to the first half of the $3^{\text {rd }}$ millenium B.C.E. by [Madjidzadeh 2012], and to the second half of the $3^{\text {rd }}$ millenium B.C.E. by [Desset 2014].

This paper pertains to the distributional regularities present in the inscriptions. Some are simply a fresh presentation and interpretation of regularities found in [Desset 2014], but most of them are new. The conclusion that emerges is that the 'Geometric' writing system may be a kind of musical notation, and that $\alpha, \beta$ and $\gamma$ may be the earliest known examples of musical scores.

\section{Status of the dotted counterparts of $\square \bigcirc \Delta \cap 0$ m and reading direction for $\gamma$}

[Desset 2014] indicated that the $\alpha$ and $\beta$ tablets are to be read starting from the top right corner, and following the boustrophedon ${ }^{2}$ principle. If they are read in this way, their contents exhibit structures and repetition of sequences of signs; conversely if they are read otherwise, no regularity is evident in the contents. So the boustrophedon reading of $\alpha$ and $\beta$ is well established.

[Desset 2014] also notes that no sign is repeated twice in succession (the 'no-sign-repetition' principle). This observation is more important than it seems, given the limited number of different signs in the system. It can be noted that this remains true if dotted signs are considered variants of dotless ones, and for instance there is no $\square \square$ sequence. This leads to the inference that dotted signs are indeed variants of their (more frequent) dotless counterparts.

1 I wish to thank Noreen Blanluet for her diligent proofreading of this paper.

2 In boustrophedon writing, the direction is reversed with each new line, like an ox ploughing a field, so that there is no 'carriage return'. 


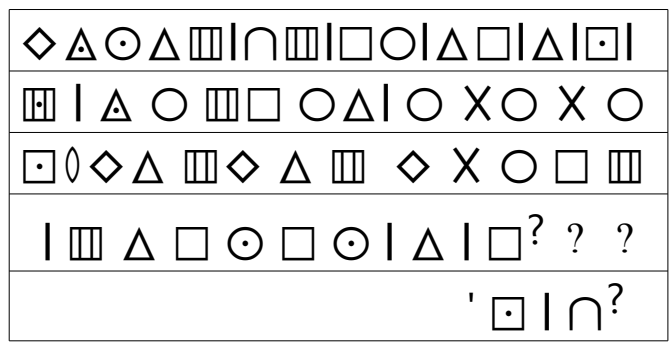

Figure 1: the $\gamma$ inscription as it appears on the tablet ${ }^{3}$ (question marks indicate tentative reading or missing signs)

Evidence points to the use of the boustrophedon principle in the $\gamma$ inscription:

- As noted in [Desset 2014], it can be assumed that the last line is to be read from right to left, and if the boustrophedon was used, then the text is to be read from the top right hand corner, exactly as in the two other tablets.

- If $\gamma$ is read from the top right corner, there is a 7-sign-long correspondence with $\alpha$ (I $\square \Delta \mathrm{IO} \square \mathrm{l}$ present in $\gamma$ on line 1 , and in $\alpha$ on three occurences).

- If $\gamma$ does not use the boustrophedon, then it violates the 'no-sign-repetition' principle:

$\circ \quad$ if all lines are read from right to left, there is a $\amalg$.

- if all lines are read from left to right, there is a $\mathrm{W} \mid \mathbf{l}$ succession between lines 3 and 4 (see part 2 below on the status of the vertical stroke).

Therefore, it seems reasonable to think that $\gamma$ is to be read beginning in the top right corner, following the boustrophedon principle, exactly like $\alpha$ and $\beta$.

From this point on, for ease of reading, all inscriptions presented will be transposed from their original direction on the tablet, in order to be read from left to right in this text.

\section{Status of the vertical stroke}

The vertical stroke sign has the following unique distributional properties, despite being the most frequent sign in the corpus:

- P1: it is the only sign appearing in all three inscriptions that also has no dotted counterpart (other never dotted signs appear in only one or two of the inscriptions);

- P2: the 'no-sign-repetition' principle remains true even if the vertical stroke is ignored, i.e. there is no $\mathrm{S} \mid \mathrm{S}$ sequence where $\mathrm{S}$ is any sign or its dotted variant. This property is significant, because there are a number of such symetrical triplets, such as $\diamond \diamond \diamond, \bigcirc X \bigcirc$, and so forth, in tablets $\beta$ and $\gamma$ (16 in $\beta, 10$ in $\gamma$, none in $\alpha$-it might be related to the absence of dotted signs in this tablet). In all such triplets, the middle and edge signs may be dotless or dotted (including the 'irregular' $\Delta \odot \Delta$ triplet in $\gamma$ ).

3 A part of this tablet is missing. On line 4, a partly legible sign is probably $\square$ or $\square$. The remaining space on this line could carry 2 or 3 signs. On line 5, the partly legible sign might be $\cap$ or $\cap$. 
- P3: distribution of the vertical stroke; below is a table showing the distribution of signs preceding and following the vertical stroke, compared to their distribution in the corpus of three tablets.

\begin{tabular}{|c|c|c|c|}
\hline Sign & $\begin{array}{r}\mathrm{Nb} \text {. of occ. in } \\
\text { the corpus }\end{array}$ & $\begin{array}{l}\text { Nb. of occ. } \\
\text { before "|" }\end{array}$ & $\begin{array}{l}\text { Nb. of occ. } \\
\text { after "|" }\end{array}$ \\
\hline$\square / \square$ & 42 & 11 & 10 \\
\hline$\bigcirc / \odot$ & 43 & 7 & 16 \\
\hline$\Delta / \Delta$ & 34 & 14 & 11 \\
\hline 四 / 龱 & 30 & 3 & 10 \\
\hline$\cap / \cap$ & 11 & 6 & - \\
\hline $0 / \theta$ & 6 & 1 & - \\
\hline$\diamond$ & 15 & 3 & - \\
\hline$\wedge$ & 6 & - & 2 \\
\hline$X$ & 5 & - & - \\
\hline$\Delta M$ & 3 & 2 & - \\
\hline$M$ & 2 & - & - \\
\hline$\forall$ & 2 & - & - \\
\hline
\end{tabular}

Figure 2: distribution of signs relative to the vertical stroke

P2 could be used to argue that the vertical stroke acts as a separator, but this runs into several counterarguments:

- If | were a separator, then 'unit' length would range from 1 sign to 18 in $\gamma$, and from 2 signs to 28 in $\beta$. This seems extremely unusual for any type of written unit, be it word, phrase, or sentence.

- Tablets $\alpha$ and $\gamma$ (if read from the top right corner) begin with a vertical stroke. Maybe these tablets are parts in a sequence of tablets, but the very regular internal structure of $\alpha$ (see below) would argue against this hypothesis. Tablet $\beta$ begins with $\diamond$, which is never preceded by the vertical stroke in the corpus (15 occurences).

- No tablet ends with the vertical stroke. In fact, in $\gamma$ on line 5, between the geometric script and the Linear Elamite inscription, the separator is a short high stroke. If the vertical stroke were a separator in Geometric writing (as it is thought to be in Linear Elamite), this place would seem quite natural for it.

Therefore, it may be postulated that the vertical stroke has a very particular and unique status in the system. It may modify the sign it precedes, which would corroborate P2. However it is unlikely to be merely a separator.

In the table illustrating $\mathrm{P} 3$ it appears that $\cap / \cap$ and $\diamond$, though relatively frequent, never follow the vertical stroke. This may be significant, like the fact that $\diamond$ is never dotted in the corpus. 


\section{Structure of the $\alpha$ inscription}

[Desset 2014] noted that $\alpha$ exhibits a multi-layered repetition structure. Here follows a different and perhaps more revealing analysis.

Below is one possible way of writing down $\alpha$ (left to right):

\begin{tabular}{|c|c|c|}
\hline $\mathrm{I} \square \Delta$ & $10 \square$ & $\mathrm{I} \triangle \square \mathrm{O}$ \\
\hline $\mid \square \Delta$ & $10 \square$ & $\mathrm{I} \triangle O \square \triangle$ \\
\hline IO四 $\square \cap \Delta$ & $10 \square$ & $\mathrm{I} \Delta \square \mathrm{O}$ \\
\hline $\mathrm{I} \square \Delta$ & $10 \square$ & $\mathrm{I} \triangle \mathrm{O} \square \Delta$ \\
\hline IO四 $\square \cap \Delta$ & $\mathrm{Im} \cap$ & $\mathrm{I} \Delta \wedge \mathrm{W}$ \\
\hline $\mathrm{I} \wedge \circlearrowleft \cap \Delta$ & $\mathrm{Im} \cap$ & $\mathrm{IO} \Delta \wedge 0$ \\
\hline $\mathrm{I} \square \Delta$ & $\mathrm{I} \mid \mathrm{D} \cap$ & $\mathrm{I} \triangle \wedge \mathrm{W}$ \\
\hline$I \wedge \circlearrowleft \cap \triangle$ & $\mathrm{I} \mid \mathrm{D} \cap$ & $10 \Delta \wedge 0$ \\
\hline
\end{tabular}

Figure 3: the $\alpha$ inscription

This exhibits a structure of 8 'lines', each consisting of a starting segment, a middle segment and an ending segment. It can be represented in a more schematic way using the following notations ${ }^{4}$ :

\begin{tabular}{|c|c|c|}
\hline $\mathrm{A} 1=\mathrm{I} \square \Delta$ & $\mathrm{A} 2=\mathrm{IO} \square \square \cap \triangle$ & $\mathrm{A} 3=\mathrm{I} \wedge \circlearrowleft \cap \triangle$ \\
\hline $\mathrm{B} 1=\mathrm{IO} \square$ & $\mathrm{B} 2=\mathrm{Im} \cap$ & \\
\hline $\mathrm{C} 11=\mathrm{I} \Delta \square \mathrm{O}$ & $\mathrm{C} 12=\mathrm{I} \triangle \mathrm{O} \square \Delta$ & $<\mathrm{C} 11$ and $\mathrm{C} 12$ always after $\mathrm{B} 1$ \\
\hline $\mathrm{C} 21=\mathrm{I} \triangle \wedge \mathrm{W}$ & $\mathrm{C} 22=10 \Delta \wedge 0$ & $<\mathrm{C} 21$ and $\mathrm{C} 22$ always after B2 \\
\hline
\end{tabular}

Figure 4: notations for the analysis of $\alpha$

So $\alpha$ can be schematically represented as:

$\left.\begin{array}{llll|}\left.\begin{array}{llll}\text { A1-B1-C11 } & \text { L1 } & & \\ \text { A1-B1-C12 } & \text { L2 } & \text { Q } 1 ~ & \\ \text { A2-B1-C11 } & \text { L3 } & & \\ \text { A1-B1-C12 } & \text { L4 } & \text { S2 } & \\ \text { A2-B2-C21 } & \text { L5 } & & \\ \text { A3-B2-C22 } & \text { L6 } & & \\ \text { A1-B2-C21 } & \text { L7 } & & \\ \text { A3-B2-C22 } & \text { L8 }\end{array}\right\} \text { Q4 }\end{array}\right\}$ H2

Figure 5: a schematic representation of $\alpha$

4 Other notations are possible, such as $A 1=|\square, B 1=\Delta I O \square| \Delta$, etc. but the analysis presented below remains the same. With the notations chosen here, all segments begin with the vertical stroke, exemplifying its peculiar status in the system; and a sequence $\mathrm{A} 1 \mathrm{~B} 1 \mathrm{~B} 2$ is also found in the tablet $\gamma$. 
It shows a structure of variation within repetition, on three levels:

- $\quad \mathrm{L} 1$ (A1-B1-C11 = I $\square \Delta \mathrm{IO} \square \mathrm{I} \triangle \square \mathrm{O}$ ) is the basic pattern.

- On the first level, L2 is formed by a variation of the ending of L1. L1+L2 add up to Q1.

- On the second level, Q2 is formed by a different variation of the beginning of Q1, thus resulting in the first half $\mathrm{H} 1$.

- If the exact same procedure were applied to the basic pattern A3-B2-C21, the result would be the following second half $\mathrm{H} 2^{\circ}$ (assuming some sort of cyclic group in variations: A1 becomes A2, A2 becomes A3, A3 becomes A1) :

$$
\begin{aligned}
& \text { A3-B2-C21 } \\
& \text { A3-B2-C22 } \\
& \text { A1-B2-C21 } \\
& \text { A3-B2-C22 }
\end{aligned}
$$

- On the third level, $\mathrm{H} 2$ is formed of a minimal variation of the beginning of this hypothetical second half $\mathrm{H} 2^{\circ}$.

So the whole $\alpha$ text can be generated with only two basic patterns (A1-B1-C11, and A3-B2$\mathrm{C} 21$ which is not directly attested) and small variations on three subsequent levels. This kind of variation within repetition is reminiscent of musical composition.

\begin{tabular}{|c|c|c|c|}
\hline$I \square \Delta$ & $10 \square$ & $\mathrm{I} \Delta \square \mathrm{O}$ & L1 \\
\hline $\mathrm{I} \square \Delta$ & $10 \square$ & $\mathrm{I} \triangle O \square \Delta$ & L2 \\
\hline$I \square \Delta$ & $10 \square$ & $\mathrm{I} \Delta \square \mathrm{O}$ & $\mathbf{L 3}$ \\
\hline $\mid \square \Delta$ & $10 \square$ & $\mathrm{I} \triangle \bigcirc \square \Delta$ & L4 \\
\hline IO四 $\square \cap \triangle$ & $\mathrm{Im} \cap$ & $\mathrm{I} \Delta \wedge \mathrm{m}$ & L5 \\
\hline $\mid \wedge \circlearrowleft \cap \Delta$ & $\mathrm{Im} \cap$ & $1 O \Delta \wedge 0$ & L6 \\
\hline $\mathrm{IO}$ & $\mathrm{Im} \cap$ & $\mathrm{I} \Delta \wedge \mathrm{W}$ & L7 \\
\hline $\mathrm{I} \wedge \circlearrowleft \cap \Delta$ & $\mathrm{Im} \cap$ & $1 O \Delta \wedge 0$ & L8 \\
\hline
\end{tabular}

Another, simpler way of viewing it is that the text is made up of two halves, each consisting of two identical pairs of lines, and then the first segments of the third line of each half are switched. This is shown in figure 6 .

The segments highlighted in yellow are switched in the actual inscription. 


\section{Structure of the $\beta$ inscription}

This inscription is structured in pairs of sequences, as noted in [Desset 2014], often with variations. Below is a representation which puts all variations at the beginning of lines:

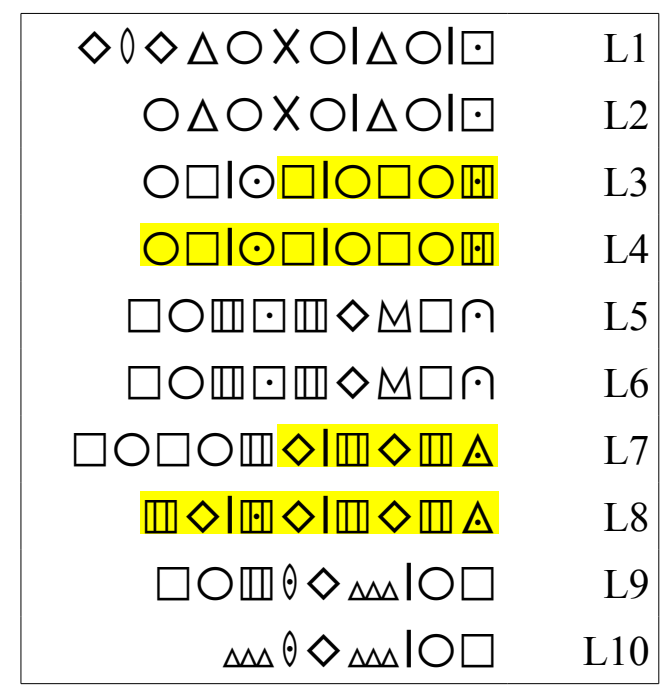

Figure 7: the $\beta$ inscription

The sequences highlighted in yellow exhibit the following interesting property: each sign of the upper sequence matches a sign of the lower sequence, while conserving the vertical strokes and the dots.

\begin{tabular}{|l|r|r|r|r|r|r|}
\hline & 1 & 2 & 3 & 4 & 5 & 6 \\
\hline Upper sequence: & $\square$ & I & $\bigcirc$ & $\amalg$ & $\odot$ & $(\mathbb{W})$ \\
\hline matches lower sequence: & $\diamond$ & I & 山 & $\triangle$ & 龱 & $(\Delta)$ \\
\hline
\end{tabular}

Figure 8: matching signs between the highlighted sequences in $\beta$. Column 6 is not attested, but inferred from columns 3, 4 and 5.

This 13-sign-long match (not counting vertical strokes) cannot be random. This reveals a deep link between some signs: if the lower sequence is the result of a transformation (perhaps a

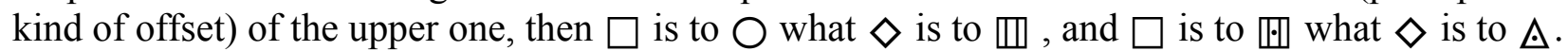
This property would be expected from transposition on a musical scale: $\mathrm{A}$ is to $\mathrm{B}$ what $\mathrm{C}$ is to $\mathrm{D}$ (on the major scale), and so forth.

The transformation is not reflexive: $\odot$ gives $\amalg$ but $\amalg$ gives $\Delta$, which is consistent with an offset on a scale. 


\section{Structure of the $\gamma$ inscription}

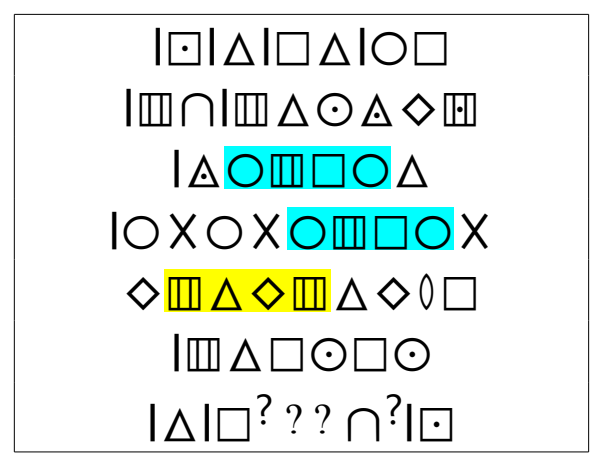

Figure 9: the $\gamma$ inscription (assuming a boustrophedon reading, see part 1).

Line breaks are arbitrary.

This inscription is far less regular than $\alpha$ or $\beta$, but it contains a few remarkable sequences:

- The longest repeating sequence is highlighted in blue in figure 9 above: $\bigcirc \square \square \bigcirc$.

- This sequence matches another sequence in $\gamma(\mathbb{m} \Delta \diamond \mathbb{W}$, highlighted in yellow) through the same transformation mentioned in the analysis of $\beta$.

- Maybe less significantly, the sequence $\diamond \mathbb{W} \Delta \diamond \mathbb{W} \Delta$ is a redoubling of $\diamond \mathbb{W} \Delta$, which matches $\square \mathrm{OW}$ through the abovementioned transformation. $\square \mathrm{OW}$ occurs four times in $\beta$, but is absent from $\alpha$ and $\gamma$.

\section{Conclusion}

Here have been presented several observations supporting the view that $\alpha, \beta$ and $\gamma$ probably denote music. The 'Geometric' writing system could be a kind of musical notation, or at least a shorthand notation for an activity related to music, such as singing or dancing.

This is consistent with the size of the sign inventory, consisting of 12 signs plus two modifiers (the dot and the vertical stroke). Such a limited inventory, and the very high frequency of $\square, \bigcirc, \Delta$, and $\square$ in the corpus, rules out a linguistic usage.

It may be that very simple geometric shapes were used because musicians needed a specific system, easily readable from a distance, with tablets inscribed on one side, so that they could be laid in front of them while playing. This would also explain why the tablets were baked: for the longterm preservation of musical scores.

Blind musicians were highly regarded in ancient Mesopotamia, as well as in other ancient cultures (see [Radner \& Robson 2011, p. 294] and [Stol, Richardson-Hewitt \& Richardson 2016, p. 362]). If we assume this remains true for Elam, the geometric shapes and boustrophedon writing may have been used to enable blind musicians to use the tablets. Boustrophedon writing and very basic shapes were also used in James Hatley Frere's and William Moon's reading systems for the blind, which look strikingly similar to the Konar Sandal 'Geometric' inscriptions. See [Johnson 1853, pp. 16-21] for details of these systems. In the same book, an advantageous feature of the boustrophedon principle for the blind is stated: "Thus the finger of the blind reader need not be removed from the paper. The place is never lost" (p. 18, emphasis in the original). 
If this were the case, the 'Geometric' inscriptions would be the earliest known form of musical notation, predating by a few centuries the Old Babylonian musical tablets dated from the beginning of the second millenium B.C.E. (see for instance [Dumbrill 2005] or [Kilmer \& Peterson 2009]). It would also be, by far, the earliest reading system for the blind. Of course, this hypothesis remains to be confirmed or disproved by further study, particularly by archaeomusicologists.

Finally, one may hope that a better understanding of the 'Geometric' script will help with the interpretation of the accompanying Linear Elamite inscriptions named B', C' and D', which could be the names of the music pieces, and with the decipherment of the Linear Elamite script.

\section{Bibliography}

Basello, Gian Pietro. (2006). The Tablet from Konar Sandal B (Jiroft) and its Pertinence to Elamite Studies (http://www.elamit.net/elam/jiroft.pdf)

Desset, François. (2014). A new writing system discovered in 3rd millennium bce iran: The konar sandal 'geometric' tablets. Iranica Antiqua. 49. 83-109. 10.2143/IA.49.0.3009239.

Dumbrill, R. J. (2005). The Archaeomusicology of the Ancient Near East. Trafford Publishing.

Johnson, E. C. (1853). Tangible Typography: or, How the blind read. London: Whitaker.

Kilmer, A. \& Peterson, J. (2009). More Old Babylonian Music-Instruction Fragments from Nippur. Journal of Cuneiform Studies, 61, 93-96.

Madjidzadeh, Y. (2012). Jiroft Tablets and the Origin of the Linear Elamite Writing System.

Cultural Relations between the Indus and the Iranian Plateau during the Third Millennium BCE, 217-43.

Radner, K. \& Robson, E.(Eds.). (2011). The Oxford Handbook of Cuneiform Culture. Oxford University Press.

Stol, M., Richardson-Hewitt, H. \& Richardson, M. (2016). Women in the Ancient Near East. Berlin, Boston: De Gruyter. 